\title{
Adopting the Chemical Potential in the High School Curriculum: Why not?
}

\author{
Paolo Lubini ${ }^{\star \star}$ and Michele D’Anna ${ }^{\mathrm{b} \star}$
}

\begin{abstract}
Discussing some examples involving the equilibrium condition in phase transitions and chemical reactions, we show how it is possible to introduce the concept of chemical potential $(\mu)$ even at the beginning of high school. This provides the students with a simple way of conceptualizing and managing quantitatively phenomena which, on the surface, are quite different; and it allows them to do this in a way which is both unifying and coherent.
\end{abstract}

Keywords: Chemical potential · Education · Equilibrium · Thermodynamics
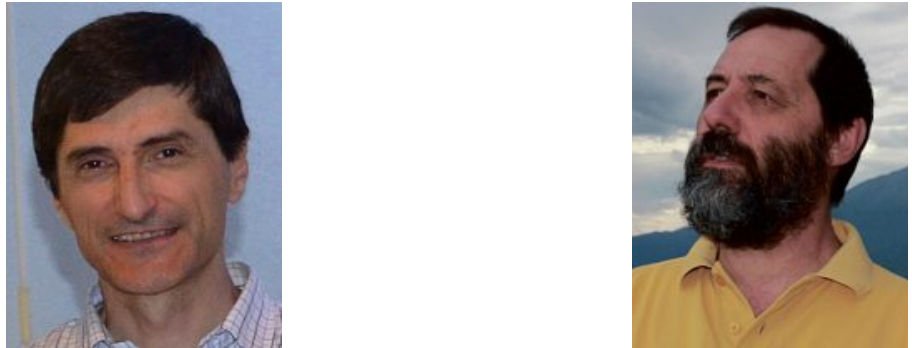

Paolo Lubini graduated in 1990 at the Swiss Federal Institute of Technology in Zurich (ETHZ) in chemistry where he also earned a PhD in 1995. After a post doc at the Georg August University of Göttingen (Germany) on X-ray structure determination of macromolecules, he has been working as a high school teacher in Locarno and in Savosa since 1997. His research interests include modeling of uniform dynamical systems as well as developing new ways of teaching chemistry based upon the early introduction of the concepts of chemical potential and entropy.

${ }^{*}$ Correspondence: Dr. P. Lubinia, M. D’Anna ${ }^{b}$

E-mail: paolo.lubini@edu.ti.ch, michele.danna@edu.ti.ch aLiceo cantonale di Lugano 2

Nuova Via San Gottardo, CH-6942 Savosa

biceo cantonale di Locarno

Via F. Chiesa 15a, CH-6600 Locarno
Michele D'Anna graduated in 1977 at the Swiss Federal Institute of Technology in Zurich (ETHZ) in theoretical physics. After being briefly active as a particle physics researcher, he has been working as a high school teacher in Locarno since 1979. During the last four decades he has been involved in several groups working on school reform; at the moment he is interested in the modernization of the basic course in physics at high school level from an interdisciplinary perspective and in the development of a modeling-based course linking physics and mathematics.

"One of the principal objects of practical research ... is to find the point of view from which the subject appears in its greatest simplicity” - J.W. Gibbs

Chemistry deals with change, and one may wonder if there is a quantity which, in some way, enables us to describe and quantify the tendency and direction of such change. Indeed there is one, the chemical potential $(\mu)$. But if we look at chemistry teaching in high school, we find no explicit trace of the chemical potential. Maybe the reason lies in the difficulties associated with this quantity, as it is introduced mainly in advanced university courses and is usually derived in terms of concepts from thermodynamics. In this contribution we would like to illustrate how this concept can be introduced in a simple way already at high school level. The power of the concept of chemical potential should become apparent if we learn to derive useful models of chemical processes by directly using this fundamental quantity rather than relying on indirect arguments. The approach discussed here has been developed largely by Job and Herrmann and has also been used in the context of a dynamical theory of heat by Fuchs. ${ }^{[1-5]}$

The basic idea is that each substance has a certain (potential) tendency to decompose or to react with other substances, to undergo a phase transition or to distribute in space. This tendency can be expressed by one and the same quantity - the chemical potential $\mu$. Yet the strength of this tendency (i.e. the numerical value of $\mu$ ) is determined by the nature of the substance as well as by its milieu, but neither by the nature of reactions partner nor the resulting products (Fig. 1 and Fig. 2).

To exemplify how it could be possible to introduce the concept of chemical potential from the beginning in order to provide a tool for the conceptualisation as well as for a unified description of chemical processes, we will present and discuss three different examples, ranging from a qualitative to a quantitative dimension.

Let us begin with water. We all know that water, at constant normal pressure, depending on temperature, will appear prevalently as a solid, as a liquid or as a vapour. By change of temperature, we can change its state. We can say that ice at room temperature experiences a driving force to melt due to a higher value of its chemical potential relative to the value of the chemical potential of liquid water. The same picture can be applied to liquid water put in a freezer where it will experience a driving force to solidify due to a difference in the values of the chemical potential. The situation can be represented graphically in a qualitative but consistent way (Fig. 3). 
Fig. 1. To evaluate if a transformation from state $A$ to state $B$ is possible, it is sufficient to compare the values of the chemical potential for the two states. Note that this gives no direct information about how long it will take for the system to go from state $A$ to state $B$.
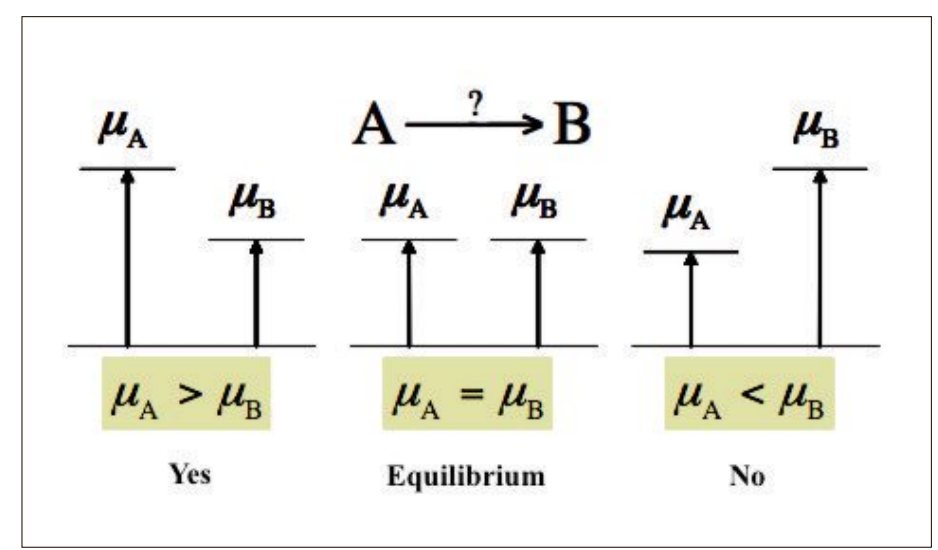

Osmosis is another well known and easy to observe phenomenon that involves water. Just consider some potatoes soaked in pure water: as time passes, one can observe that the amount of water in the potatoes increases. Again, we can conceptualise and verbalise this process using the concept of the chemical potential: there is a difference in the value of the chemical potential of water inside and outside the potatoes which results in a driving force for the process.

This experiment also suggests that dissolving salt into water causes a diminution of the value of the chemical potential of liquid water itself.

We can apply this result to the above discussion about the phase transition states of water: as can be easily seen from Fig. 4 , a decrease in the value of the chemical potential of liquid water upon addition of salt should result in a lowering of the melting temperature as well as an increase of the boiling temperature of the resulting solution. And indeed, this can be easily observed experimentally. Knowing the dependence of the value of the chemical potential from pressure, temperature and concentration, allows us not only to conceptualise and verbalise chemical phenomena, but also to make predictions which, in some cases, can be easily tested.

To further illustrate this point, let us consider another example: the temperature dependence of the vapour pressure for ethanol. The experimental setup as well as the measured data are reported in Fig. 5. In this case, each point on the plot $p$ vs T (Fig. 5, right) represents a situation of equilibrium between ethanol in the liquid $(\mathrm{EtOH}(1))$ and in the vapour phase $(\mathrm{EtOH}(\mathrm{g}))$,

$$
\mathrm{EtOH}(\mathrm{l}) \rightleftharpoons \mathrm{EtOH}(\mathrm{g})
$$

i.e. at each temperature the following equilibrium condition must be met:

$$
\mu_{\mathrm{EtOH}(1)}=\mu_{(\mathrm{EtOH}(\mathrm{g})}
$$

For a quantitative description, it is necessary to know how the values of the chemical potential of ethanol depend on pressure and temperature. Using the relations presented in Fig. 2, it is possible to set up the following equations for the values of the chemical potential of ethanol in the liquid and in the vapour phase (supposed to obey the ideal gas law):

$$
\begin{aligned}
& \mu_{\mathrm{H}_{2} \mathrm{O}(\mathrm{s})}\left(p^{\circ}, T\right)=\mu_{\mathrm{H}_{2} \mathrm{O}(1)}\left(p^{\circ}, T\right) \\
& \mu_{\mathrm{H}_{2} \mathrm{O}(\mathrm{s})}^{\circ}-\hat{s}_{\mathrm{H}_{2} \mathrm{O}(\mathrm{s})}^{\circ}\left[T-T^{\circ}\right]=\mu_{\mathrm{H}_{2} \mathrm{O}(1)}^{\circ}-\hat{s}_{\mathrm{H}_{2} \mathrm{O}(1)}^{\circ}\left[T-T^{\circ}\right]
\end{aligned}
$$

Introducing numbers (see ref. [7]) and solving for $T$ leads to a temperature of $1.5^{\circ} \mathrm{C}$ for the normal melting point of water - not that bad considering the assumptions made.

$$
\begin{aligned}
& \mu_{\mathrm{EtOH}(1)}=\mu_{\mathrm{EtOH}(1)}^{\circ}+\underbrace{\hat{v}_{\mathrm{EtOH}(1)}^{\circ}\left[p-p^{\circ}\right]}_{p \text { dependence }}-\underbrace{\hat{\hat{s}}_{\mathrm{EtOH}(1)}^{\circ}\left[T-T^{\circ}\right]}_{T \text { dependence }} \\
& \mu_{\mathrm{EtOH}(\mathrm{g})}=\mu_{\mathrm{EtOH}(\mathrm{g})}^{\circ}+\underbrace{R T \ln \left(\frac{p_{\mathrm{EtOH}(\mathrm{g})}}{p^{\circ}}\right)}_{p \text { dependence }}-\underbrace{\hat{s}_{\mathrm{EtOH}(\mathrm{g})}^{\circ}\left[T-T^{\circ}\right]}_{T \text { dependence }}
\end{aligned}
$$

By taking into account that the molar volume of a liquid is much less than the results, mathematically, in the determination of the intersection point of two straight lines, a procedure that can serve, as well, 
molar volume of a vapour (and assuming again that the vapour obeys the ideal gas law), i.e.

$$
\hat{v}_{\mathrm{EtOH}(\mathrm{l})}^{\circ}\left[p-p^{\circ}\right] \ll R T \ln \left(\frac{p_{\mathrm{EtOH}(\mathrm{g})}}{p^{\circ}}\right) \approx \hat{v}_{\mathrm{EtOH}(\mathrm{g})}^{\circ}\left[p-p^{\circ}\right] \quad=\mu_{\mathrm{EtOH}(\mathrm{g})}^{\circ}+R T \ln \left(\frac{p_{\mathrm{EtOH}(\mathrm{g})}}{p^{\circ}}\right)-\hat{s}_{\mathrm{EtOH}(\mathrm{g})}^{\circ}\left[T-T^{\circ}\right]
$$

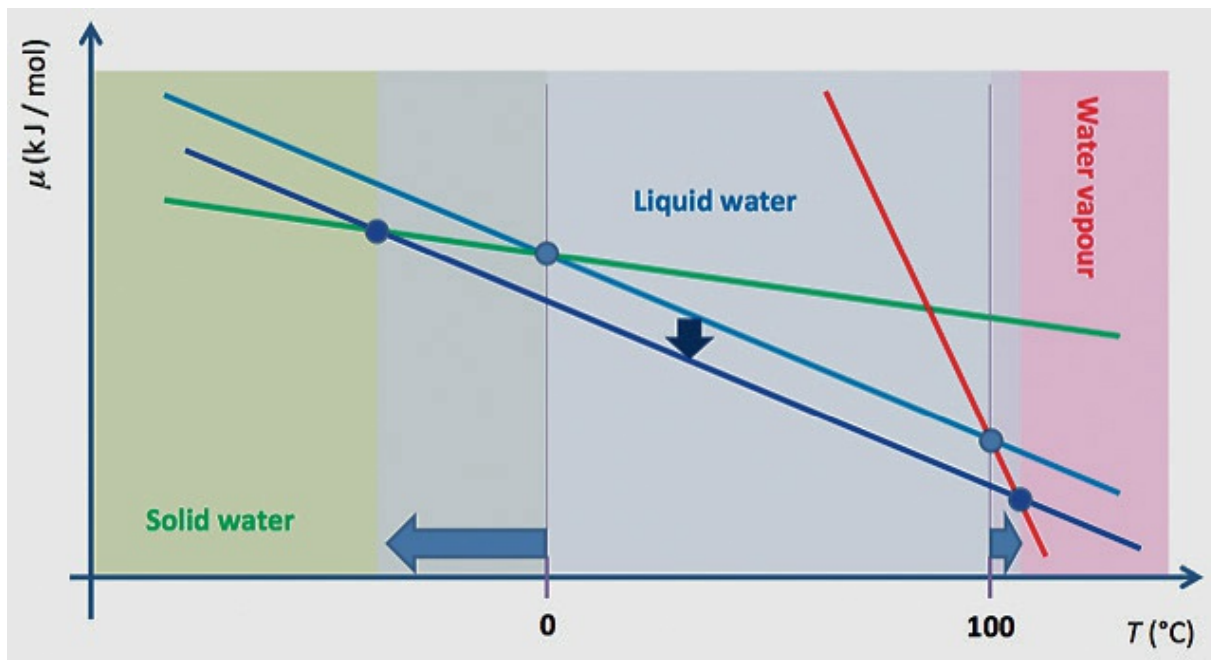

Fig. 4. Adding salt to liquid water causes a diminution of the value of its chemical potential (blue line): the resulting solution shows a lower solidification temperature and a higher boiling point.

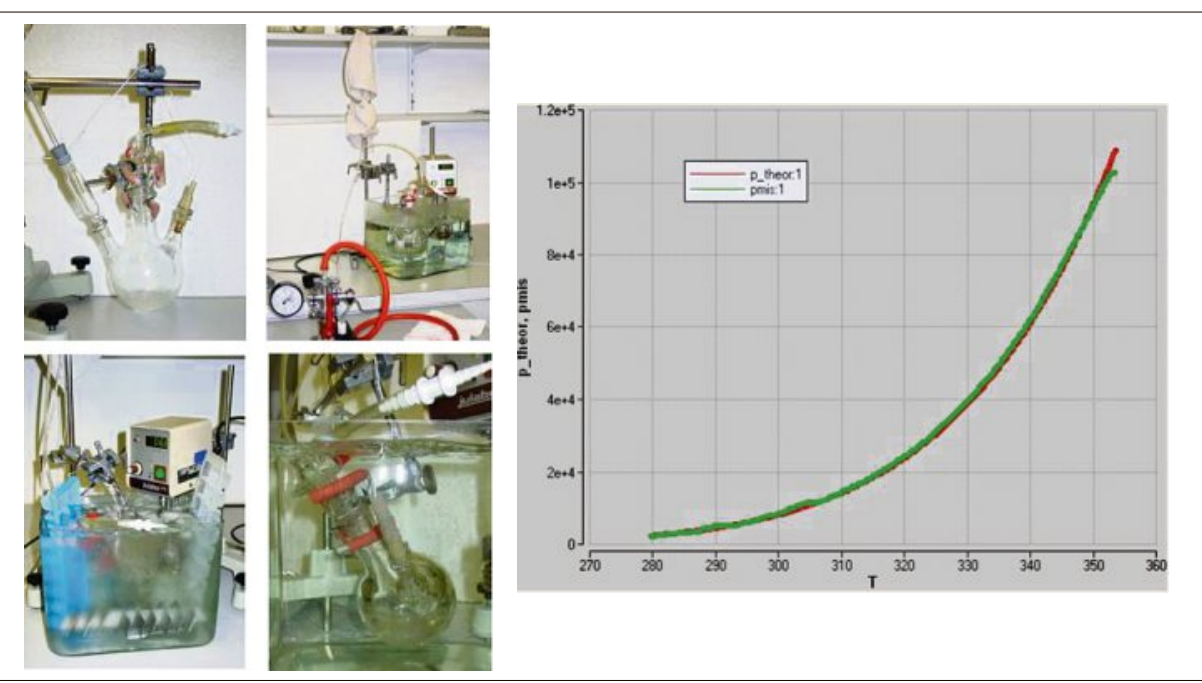

Fig. 5. Left: Experimental set-up used to measure the vapour pressure of ethanol at different temperatures. Liquid pure ethanol was put into a flask provided with sensors for temperature and pressure measurement. Air was evacuated with a vacuum pump. The flask was placed in a water bath at about $80^{\circ} \mathrm{C}$ and then progressively cooled. For the final temperature, a bath of water and ice was used. Right: The measured and the calculated values for the temperature dependence of the vapour pressure of ethanol.

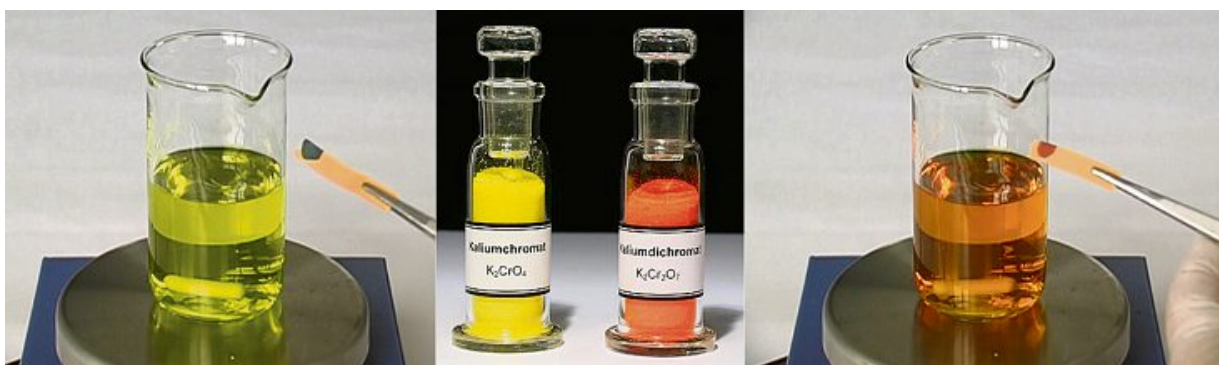

Fig. 6. Chromate/dichromate equilibrium in aqueous solution. On the left, a solution with basic $\mathrm{pH}$ where the chromate ions predominate (yellow). On the right, the same solution but this time with acidic $\mathrm{pH}$ : the dichromate ion predominates (orange).
This equation can be solved for the saturation vapour pressure of ethanol $p_{\mathrm{EtOH}(\mathrm{g})}$ yielding the well known ClausiusClapeyron relation:

$$
p_{\mathrm{EtOH}(\mathrm{g})}(T)=p^{\circ} \exp \left(-\frac{\left[T_{e b}-T\right] \Delta \hat{s}_{l \rightarrow g}^{\circ}}{R T}\right)
$$

where $\Delta \hat{s}_{l \rightarrow g}^{\circ}=\hat{s}_{\mathrm{EtOH}(\mathrm{g})}^{\circ}-\hat{s}_{\mathrm{EtOH}(\mathrm{l})}^{\circ} \quad$ corresponds to the entropy change in the phase transition under normal conditions and $T_{\text {eb }}$ is the normal boiling point of ethanol when $p_{\mathrm{EtOH}(\mathrm{g})}=p^{\mathrm{o}}$.

As can be seen from Fig. 5, right, the agreement between the measured and the calculated values for the temperature dependence of the vapour pressure of ethanol is very good.

As a final example, let us consider a chemical reaction: the equilibrium between chromate and dichromate ions in aqueous solution (Fig. 6).

This equilibrium is influenced by the $\mathrm{pH}$ and, thanks to the different colour which chromate and dichromate ions confer to the solution, is suitable for investigating how a system at equilibrium reacts to a perturbation:

$$
2 \mathrm{CrO}_{4}{ }^{2-}+2 \mathrm{H}^{+} \rightleftharpoons \mathrm{Cr}_{2} \mathrm{O}_{7}{ }^{2-}+\mathrm{H}_{2} \mathrm{O}
$$

At equilibrium, we must have:

$$
2 \mu_{\mathrm{CrO}_{4}{ }^{2-}}+2 \mu_{\mathrm{H}^{+}}=\mu_{\mathrm{Cr}_{2} \mathrm{O}_{7}{ }^{2-}}+\mu_{\mathrm{H}_{2} \mathrm{O}}
$$

If the reaction takes place at temperature and pressure under normal conditions, we only need to consider the concentration dependence of the chemical potential of the species involved. Furthermore, if the solution is sufficiently diluted, we can consider the chemical potential of water as being constant. In this way, using the relations of Fig. 2, and introducing the relative molar concentration $c_{\mathrm{A}} / c^{\mathrm{O}}{ }_{\mathrm{A}}=[\mathrm{A}]$, we get the following equilibrium equation:

$$
\begin{aligned}
& 2\left(\mu_{\mathrm{CrO}_{4}}^{\circ}{ }^{2-}+R T \ln \left[\mathrm{CrO}_{4}{ }^{2-}\right]\right)+2\left(\mu_{\mathrm{H}^{+}}^{\circ}+R T \ln \left[\mathrm{H}^{+}\right]\right) \\
& =\mu_{\mathrm{Cr}_{2} \mathrm{O}_{7}{ }^{2-}}^{\circ}+R T \ln \left[\mathrm{Cr}_{2} \mathrm{O}_{7}{ }^{2-}\right]+\mu_{\mathrm{H}_{2} \mathrm{O}}^{\circ}
\end{aligned}
$$

\section{Setting:}

$$
\Delta \mu_{R}^{\circ}=\left(\mu_{\mathrm{Cr}_{2} \mathrm{O}_{7}{ }^{2-}}^{\circ}+\mu_{\mathrm{H}_{2} \mathrm{O}}^{\circ}\right)-\left(2 \mu_{\mathrm{CrO}_{4}{ }^{2-}}^{\circ}+2 \mu_{\mathrm{H}^{+}}^{\circ}\right)
$$

and rearranging, we eventually get the well-known law of mass action:

$$
K_{e q}=e^{\frac{-\Delta \mu_{R}^{\circ}}{R T}}=\frac{\left[\mathrm{Cr}_{2} \mathrm{O}_{7}{ }^{2-}\right]}{\left[\mathrm{CrO}_{4}{ }^{2-}\right]^{2}\left[\mathrm{H}^{+}\right]^{2}}
$$


It is possible to perturb the above equilibrium not only by changing the $\mathrm{pH}$ of the solution, but also by adding water. If we add water to a solution where the dichromate ions prevail (orange), we can observe that the colour changes from orange to yellow, which suggests a shift to the left of the above equilibrium. How is it possible to conceptualize and describe this process with the chemical potential? Before adding water, we can reasonably assume that the system is at equilibrium. This means that:

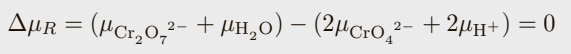

By adding water, we change the values of the chemical potential of the ions in the solution (the concentration of each ion is affected in the same way). Because of the stoichiometry of the reaction, we go from a situation where $\Delta \mu_{R}=0$ to a new one where $\Delta \mu_{R}>0$ This means that adding water builds up a chemical driving force which promotes, in this situation, the reverse reaction, in order to achieve a new equilibrium situation where, again, $\Delta \mu_{R}$ $=0$. Since the reaction is rapid enough, we do indeed observe an immediate change of colour from orange to yellow. Fig. 7 summarizes the possible effects by the perturbation of a chemical equilibrium.

\section{Conclusions}

These three examples (many more can be found in the cited literature) suggest that we can conceptualise very different chemical processes by introducing a single unifying concept, namely the chemical potential. In so doing, we achieve two goals: not only do we offer the students a solid and persistent conceptual framework, but we also reduce the number of concepts they have to deal with.

In addition, we would like to suggest an application not treated here which deserves

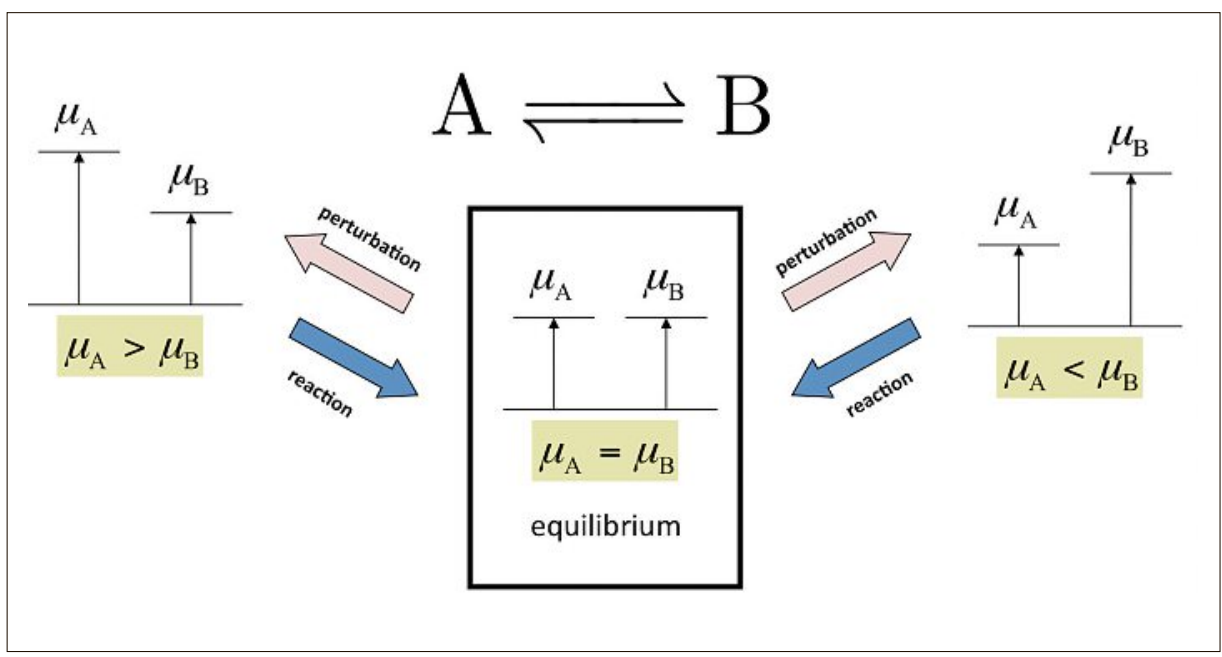

Fig. 7. From $\Delta \mu_{R}=0$ to $\Delta \mu_{R}=0$. A perturbation of an equilibrium is effective only if we go from a situation where $\Delta \mu_{R}=0$ to a new one where $\Delta \mu_{R} \neq 0$. This then results in a driving force which brings the system to a new equilibrium state promoting either the direct (if $\Delta \mu_{R}<0$ ) or the reverse reaction (if $\Delta \mu_{R}>0$ ) until $\Delta \mu_{R}=0$ again.

mention. The idea of a difference of potential as a driving force for a process as well as the introduction of combined potentials such as, for example, electro-chemical potential or gravito-chemical potential, is a fruitful one which allows us to build analogies between different area of the sciences beyond chemistry and, ranging from physics to biology, promote interdisciplinary projects as well as a coherent and unifying view of natural phenomena which consolidates gradually. ${ }^{[8]}$

Unfortunately, despite its usefulness, there seem to be a resistance toward the early introduction of the chemical potential at high school. Maybe it is only a matter of tradition or of teacher training (teachers are known to be more conservative than conservation laws ${ }^{[9]}$ ) or just of the lack of suitable school manuals. Nevertheless, it would be interesting and rewarding to pursue a didactical research project on this fascinating topic.

Received: September 11, 2017
[1] G. Job, F. Herrmann, Eur. J. Phys. 2006, 27, 353.

[2] G. Job, PdN-PhiS. 2005, 54(3), 32.

[3] G. Job, R. Rüffler, 'Physical Chemistry from a Different Angle', Springer, 2016.

[4] F. Herrmann, 'Der Karlsruher Physik-Kurs. Sekundarstufe 1 (3 Bände)' Universität Karlsruhe and Aulis-Verlag, Köln, 1999.

[5] H. U. Fuchs, 'The Dynamics of Heat - A Unified Approach to Thermodynamics and Heat Transfer', $2^{\text {nd }}$ Ed., Springer, 2010.

[6] M. D'Anna, G. Laffranchi, P. Lubini, 'Strumenti per l'insegnamento interdisciplinare della termodinamica nelle scienze sperimentali, Volume I - Il quadro concettuale', DECS, Bellinzona, 2011, http://www.scuoladecs.ti.ch/StrIIT2011/ scarica/StrIIT_11.pdf, accessed August 2017.

[7] G. Job in 'Reaktionen in Physik, Chemie und Biologie. Heft 4', Eds. G. Falk, F. Herrmann, Schroedel, 1981, p. 95.

[8] M. D'Anna, H. U. Fuchs, P. Lubini, 'System dynamics modeling: from mechanics to chemistry - A dynamical model of mutarotation of glucose', in Proc. 2008 GIREP-EPEC Conference, Cyprus, 2008.

[9] M. Alonso, Am. J. Phys. 1994, 62, 13 